\title{
HBME-1 y ciclina D 1: marcadores diagnósticos de carcinoma folicular del tiroides
}

\author{
Leonor Moyano $\mathbf{S}^{1}$, Carmen Franco $\mathrm{T}^{2}$, Laura Carreño $\mathrm{T}^{1}$, \\ Patricia Robinson Ma, Gabriel Sánchez J ${ }^{b}$. \\ HBME and cyclin D 1 as diagnostic
markers for follicular thyroid carcinoma
}

Background: Follicular carcinomas account for $15 \%$ of thyroid malignant tumors. The differential diagnosis between adenoma and minimally invasive follicular carcinoma is difficult and lacks reproducibility especially in frozen sections. As the diagnosis depends on finding foci of capsular invasion, multiple sections must be examined. Numerous immunohistochemical markers have been studied for determining malignancy. Aim: To assess the efficacy of HBME-1 and Cyclin-D1 as diagnostic markers for follicular thyroid carcinoma. Material and method: We evaluated retrospectively 21 thyroidectomy specimens of 18 women and 3 men with diagnosis of adenoma or follicular carcinomas, both by hematoxylin and eosin stain and by immunohistochemistry using the avidin biotin method for the markers HBME-1 and Cyclin D1. Results: The sensitivity and specificity of HBME-1 for the diagnosis of follicular thyroid carcinoma, were $88.9 \%$ and $100 \%$, respectively whereas for Cyclin D1 the sensitivity and specificity were $22.2 \%$ and $100 \%$, respectively. There were no false positive cases. Conclusions: HBME-1 has excellent sensitivity and specificity for the diagnosis of follicular carcinoma (Rev Méd Chile 2004; 132: 279-84). (Key Words: Cyclin D-1; Immunohistochemistry; Thyroid neoplasms)

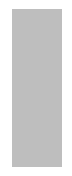

Recibido el 14 de mayo, 2003. Aceptado en versión corregida el 5 de diciembre, 2003.

${ }^{1}$ Servicio de Anatomía Patológica, Hospital Clínico Universidad de Chile, 2 Servicio de Anatomía Patológica, Hospital San Juan de Dios.

aAlumna de Medicina, Universidad de Chile

bTecnólogo Médico, Hospital Clínico Universidad de Chile

L

os carcinomas foliculares de la glándula tiroides (CFT) corresponden aproximadamente a $15 \%$ de los tumores tiroideos y su frecuencia aumenta en regiones carentes de yodo. Se presentan habitualmente como nódulos solitarios, en adultos de mediana edad y en personas añosas.

Correspondencia a: Dra. Leonor Moyano S. Servicio de Anatomía Patológica, Hospital Clínico Universidad de Chile. Santos Dumontt 999. Fono: 6788647. Fono-Fax: 7323865. E-mail: lmoyano@ns.hospital.uchile.cl
Sin embargo, se pueden ver a cualquier edad y predominan en la mujer ${ }^{1,2}$.

Los criterios diagnósticos para los carcinomas foliculares tiroideos definidos por la Organización Mundial de la Salud son: 1. Extensión neoplásica al parénquima adyacente; 2 . Invasión de vasos sanguíneos dentro o más allá de la cápsula y 3. Invasión capsular.

Este último criterio es controvertido, ya que hay discrepancia entre considerar invasión cuando hay células neoplásicas extendiéndose dentro 
de la cápsula, sin traspasarla completamente, o si se debe considerar invasión sólo cuando la cápsula se encuentra completamente transgredida por las células neoplásicas. De allí que los CFT se han clasificado en dos grupos principales: los carcinomas mínimamente invasores (CFMI) y los carcinomas invasores (CI). En ambas situaciones existe un potencial de malignidad. Sin embargo, la presencia de crecimiento invasor, no es indicador del grado de malignidad del tumor ni permite establecer pronóstico, pues se han visto carcinomas foliculares que muestran mínima invasión de la cápsula o de vasos sanguíneos que se han asociado a metástasis y, al contrario, hay carcinomas con extenso compromiso vascular o capsular con sobrevida prolongada, sin metástasis ni enfermedad recurrente por muchos años.

El diagnóstico histológico del carcinoma folicular invasor, la mayoría de las veces es claro, en cambio el mínimamente invasor representa un desafío aun en la biopsia diferida, pues el diagnóstico diferencial entre carcinoma mínimamente invasor y adenoma folicular es difícil y requiere el estudio completo de la cápsula del nódulo.

El diagnóstico de carcinoma papilar tiene criterios citológicos e histológicos característicos, que permiten establecer su identidad con valores predictivos positivos cercanos a $100 \%$ mediante citología, biopsia por aguja fina y su posterior tipificación histológica sin dificultad.

El grupo de patólogos endocrinos del Instituto de Patología de las Fuerzas Armadas de Estados Unidos de América condujo un estudio detallado de 95 casos de CFMI durante un período de 10 años. Sus resultados demostraron que los CFMI ocurren en una población más joven que otros carcinomas foliculares invasores. No hubo diferencia en la distribución por sexo, tamaño del tumor, presencia de mitosis, celularidad tumoral, oxifilia, necrosis, atipia ni volumen de tumor en la cápsula.

Las neoplasias de células de Hürtle merecen una mención especial, ya que son uno de los temas más controvertidos, especialmente desde el punto de vista pronóstico. Las neoplasias de Hürtle son aquellas conformadas en más de $75 \%$ por células foliculares oncocíticas u oxífilas. Corresponden al 2 a $3 \%$ de los carcinomas de tiroides y $20 \%$ de los carcinomas foliculares. Sin embargo, los carcinomas de células oxífilas son considerados por algunos investigadores como un subtipo de carcinoma folicular tiroideo y otros los consideran más agresivos. La sobrevida de los carcinomas foliculares de Hürtle a 15 años es de $50-60 \%$. Al parecer es similar a un carcinoma folicular no oxífilo con el mismo grado de invasión y tamaño.

Por todo lo anterior, desde el punto de vista clínico, es importante precisar el diagnóstico entre adenoma, CFMI, CFI y la variante folicular del carcinoma papilar. Este último se basa exclusivamente en el reconocimiento de los criterios nucleares a la citología. La punción por aguja fina ha demostrado ser el examen de elección como primera aproximación al diagnóstico. Los criterios para el diagnóstico de carcinoma papilar permiten un valor predictivo positivo cercano a $100 \%$. En cambio, los rangos de incertidumbre fluctúan entre 7 y $75 \%$, dependiendo de la experiencia en el método. El error diagnóstico y los exámenes no concluyentes se producen en especial por la presencia de criterios nucleares de carcinoma papilar que no son exclusivos, alrededor de $40 \%$ de las lesiones no neoplásicas y la imposibilidad de diferenciar entre adenoma y carcinoma folicular mediante el examen citológico ${ }^{3-5}$.

La literatura disponible para establecer diagnóstico y pronóstico de los carcinomas foliculares es extensa; incluye estudios inmunohistoquímicos, marcadores de proliferación, citometría de flujo, análisis cromosomal, citogenética, estudio de ploidia del ADN, análisis de actividad oncogénica, etc. Sin embargo, aún no se logra encontrar un examen que ayude claramente a determinar la conducta biológica ni el pronóstico de una neoplasia folicular en particular.

El HBME-1, desarrollado contra un antígeno desconocido de la superficie de las microvellosidades de las células mesoteliales ha mostrado reactividad en cortes histológicos de neoplasias malignas del tiroides, hasta en $56 \%$ de los casos. En una serie, 8 de 14 citologías malignas resultaron tener reactividad para HBME-1. Las 10 lesiones benignas estudiadas fueron negativas para HBME-1, demostrando no tener falsos positivos $^{8}$.

En la serie de Sack y cols, los casos que resultaron negativos correspondieron a casos considerados híbridos ${ }^{9}$.

Otro marcador es la ciclina D1, un regulador de la progresión del ciclo celular de G1 a S, codificado 
Tabla 1. Reactivos

\begin{tabular}{|lllll|}
\hline Reactivo & Laboratorio & & Dilución & tiempo (min) \\
\hline Ciclina D1 & Biogenex & Monoclonal & Prediluido & 120 \\
HBME-1 & Dako & Monoclonal & $1: 40$ & 25 \\
\hline
\end{tabular}

por un gen en el cromosoma 11q13. Se sobreexpresa en numerosos tumores, incluso sin que exista alteración del gen, en algunos casos. En recientes estudios se ha planteado que la ciclina D1 tiene un rol en la génesis tumoral tiroidea. Zous y cols, encontraron un aumento de 4 a 5 veces en la expresión de ciclina D1 al comparar carcinomas papilares con bocios multinodulares. Erickson y cols encontraron $18 \%$ de sobreexpresión de ciclina D1 en carcinomas foliculares de Hürtle comparado con $1 \%$ hallado en adenomas ${ }^{10}$.

El objetivo de este trabajo fue corroborar la utilidad de estos dos marcadores inmunohistoquímicos como herramienta diagnóstica de carcinoma folicular del tiroides en especial del carcinoma folicular y la variedad oxífila.

\section{MATERIAL Y MÉTODO}

Se seleccionaron 21 casos de neoplasias foliculares tiroideas, revisadas por dos patólogos. Los nódulos fueron estudiados en su totalidad, en cortes de $3 \mathrm{~mm}$ de espesor, fijados en formalina neutra al 10\%, los cortes en parafina de 4 micrones fueron motados en láminas teñidas con hematoxilina eosina. Utilizando los criterios para adenoma, CFMI, CI y de neoplasia oxífila de la Organización Mundial de la Salud.

Se realizó técnica de inmunohistoquímica en cortes de 4 micrones, obtenidos de bloques de parafina, mediante técnica de avidina biotina con recuperación de antígenos mediante microondas.

Se utilizó el anticuerpo HBME-1 de Dako, 1:40, incubado $25 \mathrm{~min}$ en buffer citrato pH6 con complejo avidina-biotina peroxidasa.

Se utilizó el anticuerpo ciclina D1 de Biogenex, prediluido, incubado por $120 \mathrm{~min}$, en buffer citrato pH6 (Tabla 1).

Se analizaron los resultados considerando positiva la tinción de membrana fina y granular citoplasmática en el caso de HBME-1 y la tinción nuclear en la ciclina D1.

Se realizaron con posterioridad desgastes múltiples de las inclusiones, en los casos con discordancia diagnóstica entre el estudio convencional y el análisis inmunohistoquímico.

\section{RESULTAdos}

De los 21 casos, 18 correspondieron a mujeres y 3 a hombres. La edad de los pacientes fluctuó entre 16 y 59 años, los pacientes con carcinoma entre 16 y 54 años y los pacientes con adenoma entre los 35 y 54 años.

Se diagnosticaron mediante el examen histológico convencional, 5 adenomas foliculares, 6 carcinomas foliculares invasores y 10 carcinomas foliculares mínimamente invasores.

14/21 pacientes tenían hiperplasia nodular y 7 tiroiditis crónica asociada.

En la muestra 14/21 casos correspondieron a neoplasias de Hürtle (Figura 1).

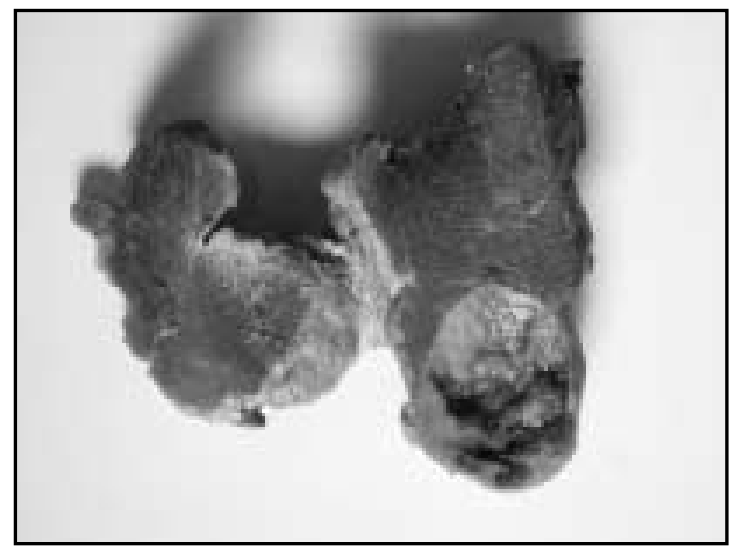

FIgURA 1. Macroscopia de neoplasia folicular oncocítica. Nódulo bien delimitado por cápsula fibrosa con área de hemorragia. 
Tabla 2. Resultados según compromiso histológico y estudio inmunohistoquímico

\begin{tabular}{|lccccccc|}
\hline Caso & $\begin{array}{r}\text { Edad } \\
\text { años }\end{array}$ & $\begin{array}{c}\text { Tamaño } \\
\text { cm }\end{array}$ & Cápsula & $\begin{array}{c}\text { Tiroides } \\
\text { Vecino }\end{array}$ & HBME1 & Ciclina D1 & Dg. \\
\hline 1 & 49 & 1,4 & + & + & + & - & Ca invasor \\
2 & - & 4,5 & + & - & + & - & CFMI \\
3 & 53 & 1,3 & + & - & \pm & - & CFMI \\
4 & 54 & 1 & - & - & - & - & Adenoma \\
5 & 35 & 2 & - & - & - & - & Adenoma \\
6 & 39 & 2,5 & + & - & + & - & CFMI \\
7 & - & 0,8 & + & + & + & - & Ca invasor \\
8 & - & 1,2 & + & + & - & - & Ca invasor \\
9 & 48 & 4,8 & + & + & + & - & Ca invasor \\
10 & 46 & 1,5 & + & + & + & \pm & Ca invasor \\
11 & 46 & 1 & + & - & + & - & CFMI \\
12 & 57 & 5 & + & - & + & \pm & CFMI \\
13 & 44 & 6,5 & + & - & + & - & CFMI \\
14 & 39 & 2,5 & - & - & - & - & Adenoma \\
15 & 55 & 2,5 & + & + & + & - & Ca invasor \\
16 & 51 & 3,5 & + & - & + & - & CFMI \\
17 & 16 & 1,8 & - & - & + & - & CFMI \\
18 & 59 & 1,5 & + & - & + & - & CFMI \\
19 & 47 & 4,0 & + & - & + & \pm & CFMI \\
20 & 47 & 3,5 & - & - & + & \pm & CFMI \\
21 & 29 & 3,0 & + & - & - & - & CFMI \\
& $16-59$ & $0,8-6,5$ & & & & & \\
\hline
\end{tabular}

CFMI: Carcinoma folicular mínimamente invasor

El tamaño de los adenomas fluctuó entre $1 \mathrm{y} 2,5 \mathrm{~cm}$ y el de los carcinomas entre $0,8 \mathrm{y}$ $6,5 \mathrm{~cm}$.

Los resultados luego del análisis inmunohistoquímico están representados en la Tabla 2. El tejido normal, con hiperplasia o tiroiditis crónica fue negativo para ambos marcadores. De las 5 lesiones previamente diagnosticadas como adenomas, 3 resultaron negativas para HBME-1, las dos lesiones positivas al ser estudiadas con numerosos desgastes a partir de sus inclusiones, revelaron un foco microscópico de invasión de la cápsula del nódulo, sin extensión al parénquima vecino (Figura 2).

Los 3 adenomas foliculares fueron negativos para ciclina D1.

Los 6 CI presentaban infiltración inequívoca a la histología corriente, $4 / 6$ fueron positivos para HBME1 (Figuras 3 y 4) y 2/6 fueron negativos. En relación a la ciclina D1 sólo 1/6 fue positivo débil, focal.

En los de 10 CFMI diagnosticados con histología corriente, 9 fueron positivos para HBME1; 6 de ellos fueron intensamente positivos, mientras que 3 fueron positivos débiles, focales. Uno de los casos fue negativo y correspondió a una variedad histológica clásica, mientras que todos los oxífilos fueron positivos.

La tinción con ciclina D1 en los carcinomas mínimamente invasores resultó negativa en 7/10 casos y positivo focal, débil en 3/10 (Figura 5).

El tejido tiroideo adyacente al tumor no presentó tinción para ciclina D1, como tampoco el tejido hiperplásico ni el con tiroiditis crónica.

En esta pequeña serie la sensibilidad del HBME-1 para el diagnóstico de carcinoma fue de $88,9 \%$ mientras que para la ciclina $\mathrm{D} 1$, fue de $22,2 \%$ 
La especificidad para ambos marcadores fue de $100 \%$. El valor predictivo positivo del HBME-1 fue de $100 \%$.

\section{DisCUSIÓN}

Los nódulos tiroideos son una patología frecuente en la población y es necesario establecer parámetros para su diagnóstico preciso y así definir su tratamiento y pronóstico.

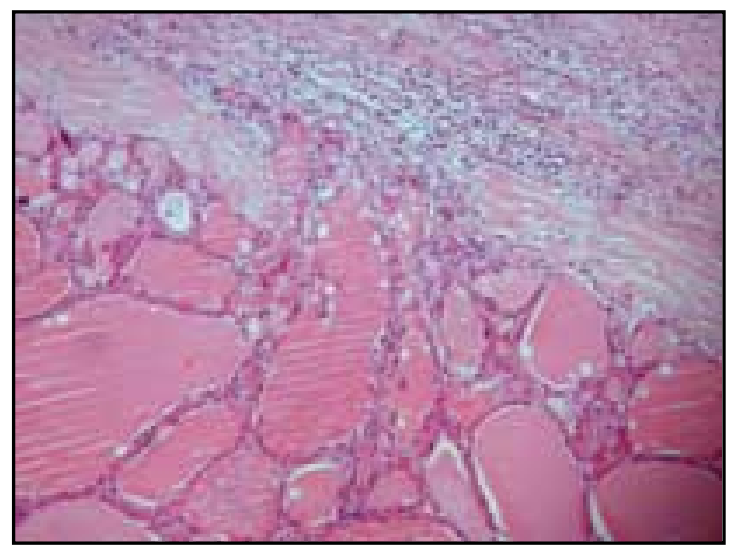

FiguRA 2. Carcinoma folicular mínimamente invasor. Proliferación folicular con compromiso capsular focal, microscópico, sin extensión al parénquima tiroideo adyacente (Hematoxilina - eosina 40x).

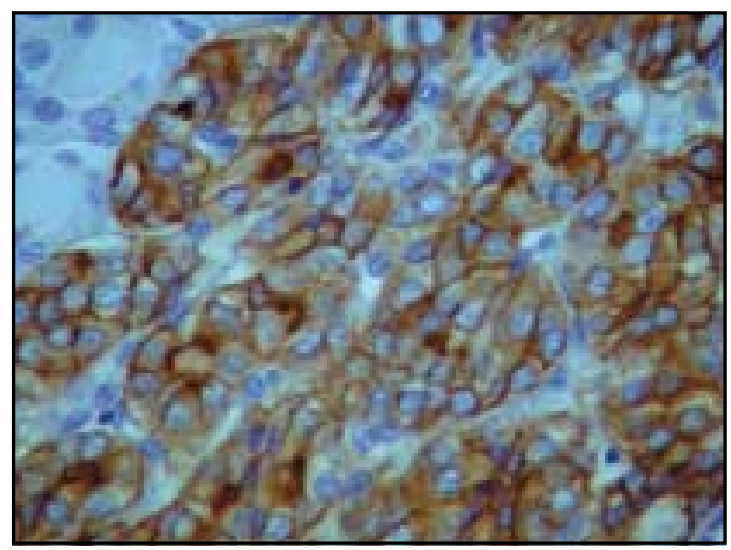

FiguRA 4. Tinción inmunohistoquímica con HBME-1 positiva en posición de membrana y citoplasmática en carcinoma folicular tiroideo (HBME-1 400x).
El diagnóstico de carcinoma papilar y la variante folicular de carcinoma papilar, en general, se establece mediante parámetros morfológicos y no requiere de técnicas complementarias salvo casos muy complejos. El valor predictivo positivo de la citología en cáncer papilar alcanza valores de 99 a 100\%.

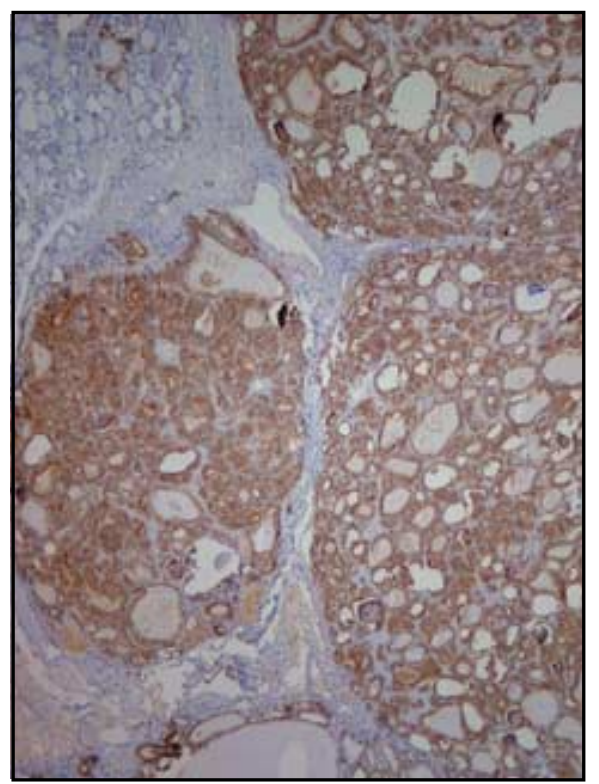

FIgURA 3. Carcinoma folicular invasor. Proliferación microfolicular con abundantes brotes neoplásicos en parénquima tiroideo más allá de la cápsula. Este caso simula hiperplasia nodular. La neoplasia es positiva para HBME-1 el tejido sano es negativo (HBME-1 40x).

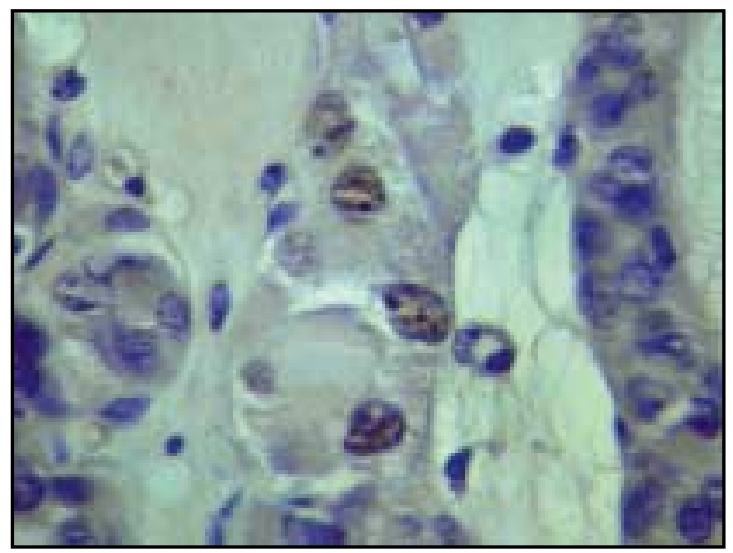

FiguRA 5. Tinción inmunohistoquímica con Ciclina D1 positiva débil y focal en posición nuclear, en caso de carcinoma folicular tiroideo (Ciclina D1 400x). 
Las neoplasias foliculares no permiten definir por citología su comportamiento biológico. Numerosos estudios no han arrojado un marcador confiable pues tienen falsos positivos en el tejido con tiroiditis vecino al tumor.

Sería deseable contar con marcadores inmunohistoquímicos específicos que, evaluados sobre el tejido morfológicamente neoplásico, nos permitan tipificar lesiones de curso maligno. En esta muestra aunque reducida, la ciclina D1 demostró ser un marcador específico en cáncer tiroideo y no presentó falsos positivos. Sin embargo, su sensibilidad es baja, 22,2\%. Por lo tanto, su utilidad es restringida para confirmar carcinoma en una biopsia diferida de compleja interpretación ni permitiría seleccionar pacientes que pudieran evitarse la cirugía al aplicar la técnica a una muestra obtenida por punción.

El marcador HBME1, también fue específico al no teñir lesiones benignas, pero éste si fue altamente sensible para pesquisar patología maligna. Una sensibilidad de $88,9 \%$ es muy deseable para elegir un marcador de utilidad para detectar

\section{REFERENCIAS}

1. Danese D, Sciacchitano S, Farsetti A. Diagnostic accuracy of conventional versus sonographyguided fine-needle aspiration biopsy of thyroid nodules. Thyroid 1998; 8: 15-21.

2. MAZZAFERRI EL. Management of a solitary thyroid nodule. New England Journal of Medicine 1993; 328: 553-9.

3. Gershergorn MC, McClung MR, Chung EW. Fineneedle aspiration citology in the preoperative diagnosis of thyroid nodules. Annals of Internal Medicine 1997; 126: 226-30.

4. La Rosa GL, Belfiore A, Giufrrida D. Evaluation of the fine-needle aspiration biopsy (FNAB) in the preoperative selection of cold thyroid nodules. Cancer 1991; 67: 2137-41.

5. Anderson JB, WebB AJ. Fine-needle aspiration biopsy and the diagnosis of thyroid cancer. British Journal of Cancer 1987; 74: 292-6. cáncer. Sobre todo si la tinción es difusa e intensa en las células en estudio.

Una ventaja importante detectada en esta muestra fue que permitió reclasificar dos lesiones calificadas mediante el estudio convencional, que consiste en el análisis histológico de un corte por cada dos o tres milímetros de tejido. Las neoplasias diagnosticadas como adenomas al realizar desgastes múltiples demostraron compromiso de la cápsula de algunos micrones, por lo que se demostró su naturaleza de CFMI.

La mayor frecuencia de tinción de carcinomas con HBME-1 demostrada en este estudio a diferencia de $56 \%$ de la literatura puede deberse a la concentración de carcinomas de Hürtle (14/18), ya que todos estos resultaron positivos y sólo dos carcinomas foliculares habituales fueron negativos.

El HBME-1 tiñe otros adenocarcinomas, por lo que la evaluación debe considerar la demostración cito-histológica que el tumor es de origen tiroideo en primer lugar.

Los resultados nos indican que una tinción fuertemente positiva con HBME-1 es altamente sugerente de carcinoma.

6. Raphael S, Apel R, Asa S. Brief report: Detection of High-Molecular-Weight cytokeratins in neoplastic and non-neoplastic thyroid tumors using microwave antigen retrieval. Modern Pathology 1995; 8: 870-2.

7. WinZer R, SCHMUTZier C, JAKOBS TJ. Reverse transcriptase-polymerase chain reaction analysis of thyrocyte-relevant genes in fine-needle aspiration biopsy of the human thyroid. Thyroid 1998; 8: 981-7.

8. Cheung C, Ezzat S, Freeman J, Rosen I, Asa S. Inmunohistochemical diagnosis of papillary thyroid carcinoma. Modern Pathology 2001; 14: 338-42.

9. SACK M, Astengo-Osuna C, Livolsi V. HBME-1 Inmunostaining in thyroid fine needle aspirations: a useful marker in the diagnosis of carcinoma. Modern pathology 1997; 10: 668-74.

10. ERICKson L, Long J, Goewner J, Lohse C. Pathologic features, proliferative activity, and cyclin D1 expression in Hürtle cell neoplasm's of the thyroid. Modern Pathology 2000; 2: 186-92. 